\title{
XMM-Newton observations of the low-mass X-ray binary XB 1832-330 in the galactic globular cluster NGC 6652
}

\author{
L. Sidoli ${ }^{1}$, N. La Palombara ${ }^{1}$, T. Oosterbroek ${ }^{2}$, and A. N. Parmar ${ }^{3}$ \\ 1 INAF, Istituto di Astrofisica Spaziale e Fisica Cosmica, via E. Bassini 15, 20133 Milano, Italy \\ e-mail: sidoli@iasf-milano.inaf.it \\ 2 Science Payload and Advanced Concepts Office, ESA, ESTEC, Keplerlaan 1, 2200 AG Noordwijk, The Netherlands \\ ${ }^{3}$ Research and Scientific Support Department of ESA, ESTEC, Keplerlaan 1, 2200 AG Noordwijk, The Netherlands
}

Received 4 February 2008 / Accepted 25 May 2008

\section{ABSTRACT}

\begin{abstract}
Context. We report on two XMM-Newton observations performed in 2006 of the luminous low-mass X-ray binary XB 1832-330, which is located in the galactic globular cluster NGC 6652 and is probably an ultracompact binary (orbital period, $P_{\text {orb }}$, of $<1 \mathrm{~h}$ ). Aims. The aim of these observations is to investigate the low-energy absorption towards XB 1832-330 and in particular to search for the Ne-rich material local to the binary, which has been suggested as a possible spectral signature of Neon-rich, degenerate companions.

Methods. XMM-Newton observed the source twice, in 2006 September and October. High resolution spectroscopy with the XMM-Newton RGS was used to estimate the ratio of the neutral neon-to-oxygen column densities to search for an anomalous Ne abundance in this X-ray binary.

Results. We find no evidence of anomalous $\mathrm{Ne} / \mathrm{O}$ ratios, finding $\mathrm{Ne} / \mathrm{O}=0.18 \pm 0.06$ and $\mathrm{Ne} / \mathrm{O}=0.17 \pm 0.03$ ( $1 \sigma$ uncertainties) in the two observations. These values are consistent with the one in the interstellar medium. Timing analysis of EPIC data suggests possible periodicities at $9170 \pm 235 \mathrm{~s}$ and $18616 \pm 531 \mathrm{~s}$, which need confirmation. A by-product of these observations consists of the spatial analysis of the source field, which resulted in the detection of 46 faint sources within the EPIC field of view, which are not present in the Second XMM-Newton Serendipitous Source Catalogue. All these faint sources are probably foreground objects.

Conclusions. We performed the first high spectral resolution observations of XB 1832-330, a probable ultracompact binary, without finding any evidence of an anomalous Ne abundance.
\end{abstract}

Key words. X-rays: individuals: XB 1832-330 - stars: neutron - X-rays: binaries - accretion, accretion disks

\section{Introduction}

XB 1832-330 was discovered using HEAO-1 (Hertz \& Wood 1985 ) and is one of the bright low-mass X-ray binaries (LMXBs) located in galactic globular clusters. The source was observed with ROSAT (Predehl et al. 1991; Johnston et al. 1996) and associated with the galactic globular cluster NGC 6652. The ROSAT uncertainty region was observed in 2000 May with the High Resolution Camera-Imager (HRC-I) camera on the Chandra X-Ray Observatory (Heinke et al. 2001). Four X-ray sources were detected within $30^{\prime \prime}$ of the cluster centre, including the bright LMXB XB 1832-330 (named "source A" or CXOGLB J183543.6-325926 in Heinke et al. 2001). The detection of a type I X-ray burst confirmed the neutron star nature of the compact object (in 't Zand et al. 1998), although one of the other fainter X-ray sources detected with Chandra could also be responsible for the burst (Heinke et al. 2001).

The significantly smaller Chandra uncertainty region allowed a secure identification of the optical counterpart (Heinke et al. 2001) with an $M_{V}=+3.7$ object (HST observations). A clear 0.08 mag variability in the $V$ and $I$ lightcurves was observed (Heinke et al. 2001), with possible periods of $\sim 3300 \mathrm{~s}$, $\sim 8000 \mathrm{~s}$ (or $\sim 16000 \mathrm{~s}$ for ellipsoidal variations), as well as nonperiodic flickering. The short temporal coverature did not allow

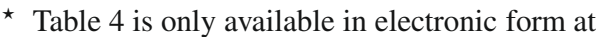
http://www . aanda.org the three periodicities to be clearly separated. Heinke et al. also notes that the optical magnitude is compatible with the donor star being in an ultracompact binary (orbital period, $P_{\text {orb }}$, less than about $1 \mathrm{~h}$ ). If one of the three periodicities is associated with the orbital period, the secondary could be a white dwarf or a low-mass M dwarf (Heinke et al. 2001).

The X-ray spectroscopy of XB 1832-330 has been performed with ASCA (Mukai \& Smale 2000) and BeppoSAX (Parmar et al. 2001) and indicates that no simple model provides acceptable fits to the spectrum, suggesting that partial covering absorption, or other spectral complexity plays an important role in modifying the X-ray emission at low energies. Another more recent observation at high energies (above $20 \mathrm{keV}$ ) has been performed with IBIS on-board INTEGRAL (Tarana et al. 2006).

The galactic globular cluster NGC 6652 is located at a distance of $9.3 \mathrm{kpc}$. It has a metallicity of $[\mathrm{Fe} / \mathrm{H}]=-0.9$ and a low reddening $\left(E_{B-V}=0.10 \pm 0.02\right)$ (Ortolani et al. 1994), which translates into an absorption, $N_{\mathrm{H}}$, of $\sim 5.5 \times 10^{20} \mathrm{~cm}^{-2}$ (Predehl \& Schmitt 1995).

We report here on the results of two XMM-Newton observations performed in order to investigate the low-energy absorption towards XB 1832-330, in particular to search for Ne-rich material local to the binary, which has been suggested as a possible spectral signature of Neon-rich, degenerate companions in binaries (Juett et al. 2001). 

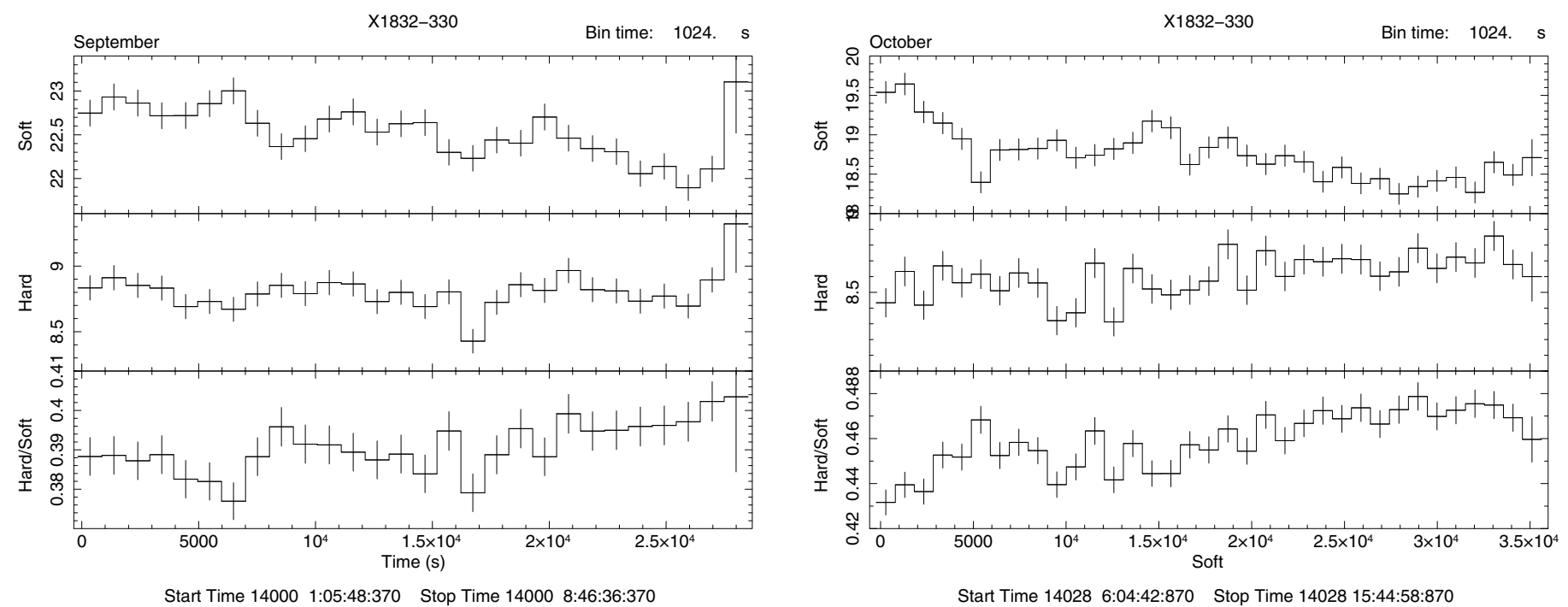

Fig. 1. PN XB 1832-330 lightcurves of the two observations in the energy ranges $0.3-2 \mathrm{keV}$ and $2-12 \mathrm{keV}$. The bottom panels show the hardness ratios (counts between $2-12 \mathrm{keV}$ divided by those between $0.3-2 \mathrm{keV}$ ) in the two observations. The binning is $1024 \mathrm{~s}$.

\section{Observations and data reduction}

XB 1832-330 was observed with XMM-Newton on 2006 September 22 (hereafter, obs I) and on 2006 October 20 (obs II), for a net exposure of $\sim 28 \mathrm{ks}$ and $\sim 34 \mathrm{ks}$, respectively (obsIDs 0400790101 and 0400790301). Data were reprocessed using version 7.1.0 of the Science Analysis Software (SAS). Known hot, or flickering, pixels and electronic noise were rejected. Response and ancillary matrix files were generated using the SAS tasks rmfgen and arfgen. The EPIC pn was operated in its Small Window mode, the MOS1 camera was in its Prime Partial Window mode, with the central CCD operated in Timing Mode and with the other CCDs in Imaging Mode. The MOS2 camera was operated in its Prime Partial Window with all the CCDs in Imaging Mode. The EPIC MOS and pn observations used the medium thickness filter. Spectra were extracted using PATTERN 0 for the MOS cameras (only single events) and PATTERNS from 0 to 4 (single and double events) in the pn. The RGS source and background events were extracted by making spatial and energy selections on the event files.

Source counts were extracted from circular regions of $40^{\prime \prime}$ radius for the pn and from a circular corona with an internal radius of $8^{\prime \prime}$ and an external radius of $1^{\prime}$ for MOS2. Source counts in MOS1 timing mode were extracted from $R A W X=309$ to $R A W X=330$, excluding the central core with an internal radius of 5 RAW pixels. The MOS1 and MOS2 data clearly suffered from the effects of pile-up. We checked iteratively with the SAS task epatplot that the events selected from annular regions in the MOS cameras were not significantly affected by pile-up after this spatial selection. Background counts were obtained from similar sized regions offset from the source positions. For the MOS1 timing mode, the background region has been taken from an outer CCD that collected data in imaging mode. The background did not show evidence of any significant flaring activity.

To ensure applicability of the $\chi^{2}$ statistics, the net spectra were rebinned such that at least 20 counts per bin were present and such that the energy resolution was not over sampled by more than a factor 3 .

\section{Analysis and results}

\subsection{Spectroscopy}

The XB 1832-330 lightcurves and hardness ratios do not show evidence for any large spectral variability (Fig. 1). We therefore extracted averaged spectra from each of the two observations, which were then analysed separately, withouth any further filtering or selection.

Since the three EPIC instruments show significant discrepancies below $0.8 \mathrm{keV}$ when they are compared, with opposite structured residuals if fitted with the same spectral model (Fig. 2), we conservatively decided to limit the spectral analysis to the range 0.8-12 keV, for both the MOS and the pn. Similar problems and discrepancies at low energies have often been observed in other X-ray binaries (e.g. Sidoli et al. 2005; Boirin et al. 2005). The softest energy range was covered with RGS1 and RGS2, which both extend down to $0.4 \mathrm{keV}$. For each observation separately, the two RGS (0.4-2 keV) spectra were fitted with the EPIC pn and the two MOS (0.8-12 keV) spectra. Free relative normalizations between the instruments were included. The net source count rates are reported in Table 1.

Since the main aim of these XMM-Newton observations was to investigate the low energy absorption towards XB 1832-330 and, in particular, the estimate of the $\mathrm{Ne} / \mathrm{O}$ ratio, we adopted a variable abundance absorption model (VPHABS in XSPEC), with the elemental abundances set to the ISM values of Wilms et al. (2000), except for those of $\mathrm{O}, \mathrm{Ne}$, and $\mathrm{Fe}$, which were set to zero. Their effect on the spectral shape has been replaced with three edges $(\mathrm{O}-\mathrm{K}, \mathrm{Fe}-\mathrm{L}, \mathrm{Ne}-\mathrm{K})$ with energies fixed at 0.543 , 0.706, and $0.869 \mathrm{keV}$ (e.g. Paerels et al. 2001) and edge depths that were allowed to vary. In this way we could also account for a local $\mathrm{Fe}$ abundance that could be different from the cosmic value.

We tried different models for the continua of the two observations. A single-component model was never able to fit the data adequately, as already demonstrated by previous observations with ASCA (Mukai \& Smale 2000) and BeppoSAX (Parmar et al. 2001). Different combinations of simple models were tried: for the soft component we adopted a blackbody and a disk blackbody model (DISKBB in XSPEC), while for the 


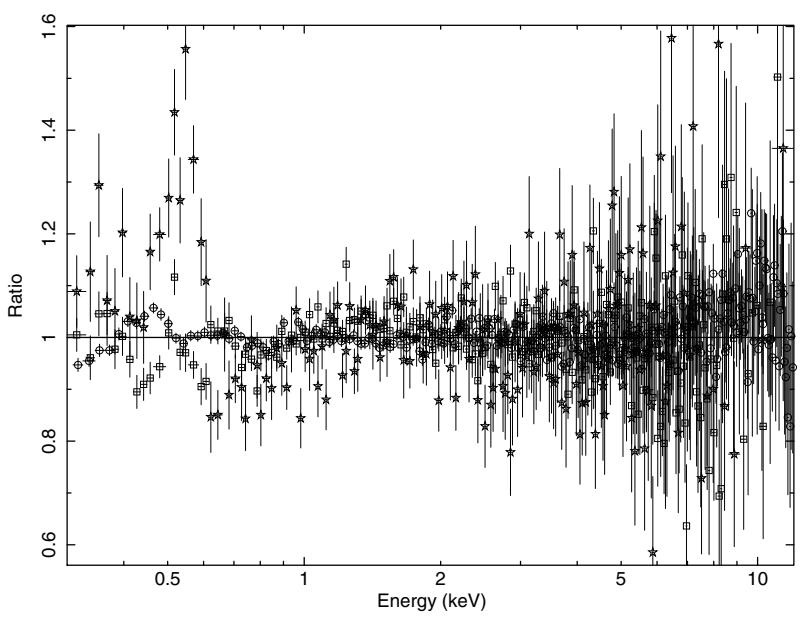

Fig. 2. Ratio between the data and the model when fitting the MOS1, MOS2, and pn spectra of obs I with a double-component continuum (power law, together with a blackbody) absorbed by a partial covering fraction component, which accounts for the global spectral shape of the X-ray emission. The different (and "opposite") residuals in the pn (open circles), compared with the MOS1 (stars) and MOS2 (squares), are evident. A similar structured excess is present in the obs II spectrum.

Table 1. Net count rates (in units of $\mathrm{s}^{-1}$ ) for XB 1832-330 during the two observations.

\begin{tabular}{lcc}
\hline \hline & Obs I & Obs II \\
\hline RGS1 & $1.113 \pm 0.007$ & $0.874 \pm 0.005$ \\
RGS2 & $1.303 \pm 0.007$ & $1.064 \pm 0.006$ \\
pn & $32.38 \pm 0.04$ & $30.33 \pm 0.04$ \\
MOS1 & $4.42 \pm 0.03$ & $3.74 \pm 0.02$ \\
MOS2 & $4.23 \pm 0.01$ & $4.11 \pm 0.01$ \\
\hline
\end{tabular}

high energy component we used a power law, a cutoff power law and a Comptonization emission model (COMPTT in XSPEC). We tried all possible combinations of these models, obtaining equally good fits and similar spectral parameters. Since there is no evidence in the XMM-Newton energy band of a cutoff (resulting in an unconstrained electron temperature in the COMPTT model or in a high-energy cutoff well above $10 \mathrm{keV}$ ), we adopted the power law plus a blackbody model for the continuum.

From the depth of the edges and the elemental cross sections, the element column densities were measured, hence the $\mathrm{Ne} / \mathrm{O}$ ratio, which has been compared to that of the interstellar medium (ISM) value of 0.18 , assuming the ISM abundances of Wilms et al. (2000). The spectral results are reported in Table 2 (for the continuum) and in Table 3 (for the edge depths, $\tau_{\text {edge }}$, columns, $N_{\mathrm{Z}}$, and equivalent hydrogen columns, $N_{\mathrm{H}}$, together with the final $\mathrm{Ne} / \mathrm{O}$ ratio, for the two observations).

We then analyzed the RGS spectra alone, to investigate the fine structure around the absorption edges more closely. We adopted the same shape for the continua as found in the broader band spectroscopy, with temperature of the blackbody component and the power-law photon index fixed to the best-fits reported in Table 2, but with all the normalizations free. The same method of measuring the edge depths of $\mathrm{Ne}, \mathrm{O}$, and $\mathrm{Fe}$ was applied to the RGS1 and RGS2 spectra (variable absorption model, together with three edges). The best fit in both the observations (reduced $\chi^{2}=1.105$ (1735 d.o.f.) and $\chi^{2}=0.937$ (2070 d.o.f.)) is obtained adding a further absorption feature, a Gaussian line at energy of $\sim 0.53 \mathrm{keV}$, which is compatible with absorption from interstellar O I and O II (see e.g. Juett et al. 2004). In
Table 2. Spectroscopy of XB 1832-330 with the same model adopted for the two observations (RGS1+RGS2+pn+MOS1+MOS2 data) consisting of a power-law model together with a blackbody, both equally absorbed by a variable absorption model, VPHABS in XSPEC.

\begin{tabular}{lcc}
\hline \hline Parameters & Obs I & Obs II \\
\hline$N_{\mathrm{H}}\left(10^{22} \mathrm{~cm}^{-2}\right)$ & $0.058 \pm 0.006$ & $0.094 \pm 0.006$ \\
$k T_{\mathrm{bb}}(\mathrm{keV})$ & $0.62 \pm 0.01$ & $0.61 \pm 0.01$ \\
$R_{\mathrm{bb}}(\mathrm{km})$ & $3.1 \pm 0.1$ & $3.5 \pm 0.1$ \\
$\Gamma$ & $1.58 \pm 0.02$ & $1.52 \pm 0.02$ \\
Flux $\left(10^{-11} \mathrm{erg} \mathrm{cm}^{-2} \mathrm{~s}^{-1}\right)$ & 1.6 & 1.5 \\
$L_{\mathrm{X}}(0.5-10 \mathrm{keV})\left(10^{36} \mathrm{erg} \mathrm{s}^{-1}\right)$ & 1.6 & 1.5 \\
red. $\chi^{2}$ (d.o.f.) & $1.154(2363)$ & $1.044(2712)$ \\
\hline
\end{tabular}

Table 3. Results on the photoelectric absorption towards XB 1832-330 (see Table 2 for the continuum emission parameters) when fitting RGS+pn+MOS spectra. Quoted uncertainties are at $1 \sigma$ confidence.

\begin{tabular}{llllll}
\hline \hline Obs & Edge & $\tau_{\text {edge }}$ & $\begin{array}{l}N_{\mathrm{Z}} \\
\left(10^{17} \mathrm{~cm}^{-2}\right)\end{array}$ & $\begin{array}{l}N_{\mathrm{H}} \\
\left(10^{21} \mathrm{~cm}^{-2}\right)\end{array}$ & $\mathrm{Ne} / \mathrm{O}$ \\
\hline I & Ne K & $0.040_{-0.010}^{+0.009}$ & $1.1 \pm 0.3$ & $1.3 \pm 0.3$ & \\
& O K & $0.345_{-0.029}^{+0.024}$ & $6.1_{-0.5}^{+0.4}$ & $1.2 \pm 0.1$ & $0.18 \pm 0.06$ \\
& Fe L & $0.084_{-0.014}^{+0.013}$ & $0.12 \pm 0.02$ & $0.45 \pm 0.07$ & \\
\hline II & Ne K & $0.055_{-0.009}^{+0.009}$ & $1.5 \pm 0.3$ & $1.7 \pm 0.3$ & \\
& O K & $0.505_{-0.025}^{+0.025}$ & $8.9 \pm 0.4$ & $1.8 \pm \mathbf{0 . 1}$ & $0.17 \pm 0.03$ \\
& Fe L & $0.107_{-0.014}^{+0.014}$ & $0.15 \pm 0.02$ & $0.57 \pm 0.07$ & \\
\hline
\end{tabular}

Figs. 3 and 4, we show both the net RGS spectra and the structure around the $\mathrm{O}$ edge. The absorption line at $0.53 \mathrm{keV}$ has a normalization of $-1.73( \pm 0.35) \times 10^{-4}$ photons $\mathrm{cm}^{-2} \mathrm{~s}^{-1}$ (Obs I, $E W=3 \pm 1 \mathrm{eV})$ and $-1.51\left(\left(_{-0.41}^{+0.39}\right) \times 10^{-4}\right.$ photons $\mathrm{cm}^{-2} \mathrm{~s}^{-1}$ (Obs II, $E W=5 \pm 1 \mathrm{eV}$ ). The resulting depths for the absorption edges are the following: $\tau_{\mathrm{O}}=0.40 \pm 0.021, \tau_{\mathrm{Ne}}=0.10_{-0.020}^{+0.018}$, $\tau_{\mathrm{Fe}}=0.16_{-0.017}^{+0.016}$ in Obs I, and $\tau_{\mathrm{O}}=0.55_{-0.018}^{+0.029}, \tau_{\mathrm{Ne}}=0.094_{-0.017}^{+0.022}$, $\tau_{\mathrm{Fe}}=0.185_{-0.016}^{+0.019}$ in Obs II (all errors on the depth of the edges are at $1 \sigma)$. The resulting ratios $\mathrm{Ne} / \mathrm{O}$ are $0.39_{-0.08}^{+0.07}(\mathrm{Obs} \mathrm{I}, 1 \sigma$ uncertainty) and $0.27_{-0.05}^{+0.06}$ (Obs II), which are within $2.6 \sigma$ and $1.8 \sigma$, respectively, so consistent with the ISM value of 0.18 . Thus we confirm with high-resolution RGS data alone, which was found during the broader band analysis, with no evidence of an unusual ratio $\mathrm{Ne} / \mathrm{O}$.

\subsection{Timing analysis}

From the Lomb-Scargle diagram of the obs I there is evidence of a periodicity near $P=9170 \pm 235 \mathrm{~s}$, with a significance of about $0.6 \%$ (see Fig. 5, left panel). Folding on this period reveals a smooth sinusodial modulation with an amplitude of $0.78 \pm 0.1 \%$ (Fig. 6). This periodicity is not the same as previously claimed values (Heinke et al. 2001), but periods in the $2-4 \mathrm{~h}$ range have been claimed before. The period is rather long compared to the observation duration, thus we cannot say much about the longterm stability. However, it is quite likely $(99.3 \%)$ that during this observation an approximately sinusodial modulation with this period was present.

In obs II there is no evidence of the $P=9170$ s periodicity. However, there is evidence of modulation with a period of $18616 \pm 531 \mathrm{~s}$. From the Lomb-Scargle periodogram this period is significant at the $0.05 \%$ level (see Fig. 5, right panel). It 

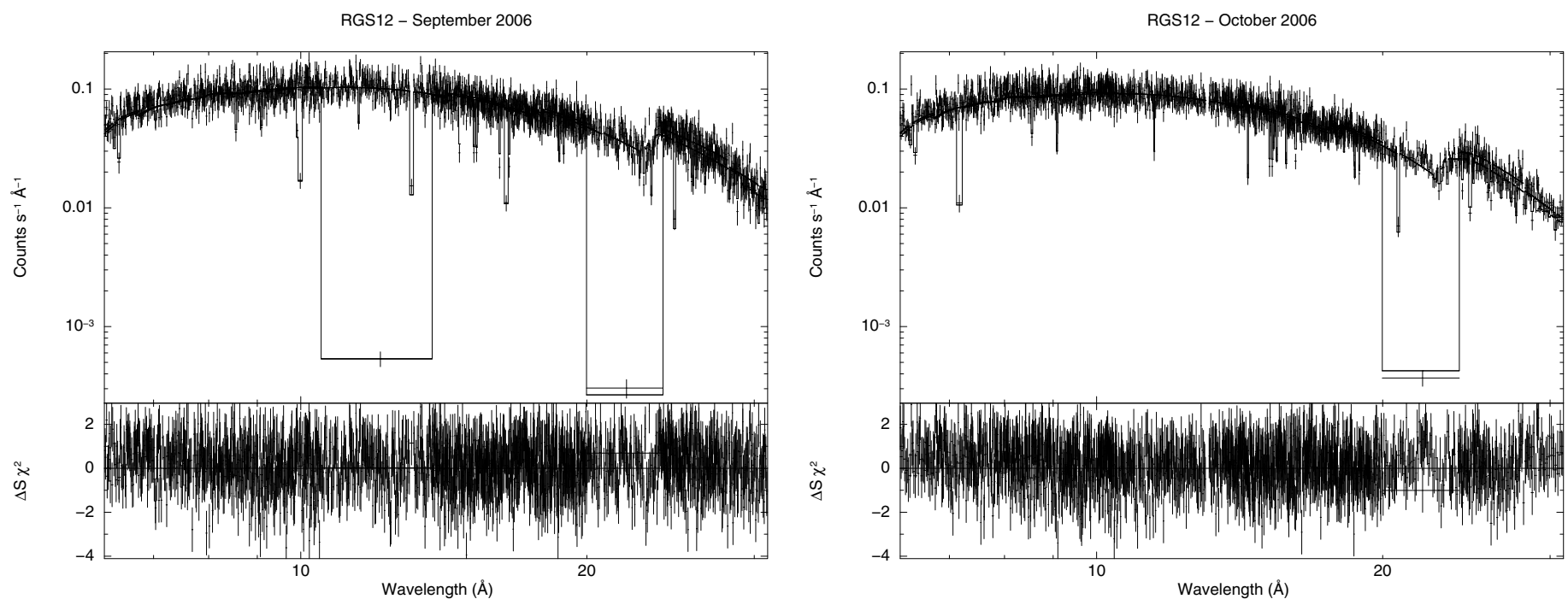

Fig. 3. RGS1 and RGS2 XB 1832-330 spectra in the two observations, fitted with the continuum model used to fit the broad-band spectra (see text). The bottom panels show the residuals in units of standard deviations. The missing regions in the RGS plots are due to the failed CCDs.
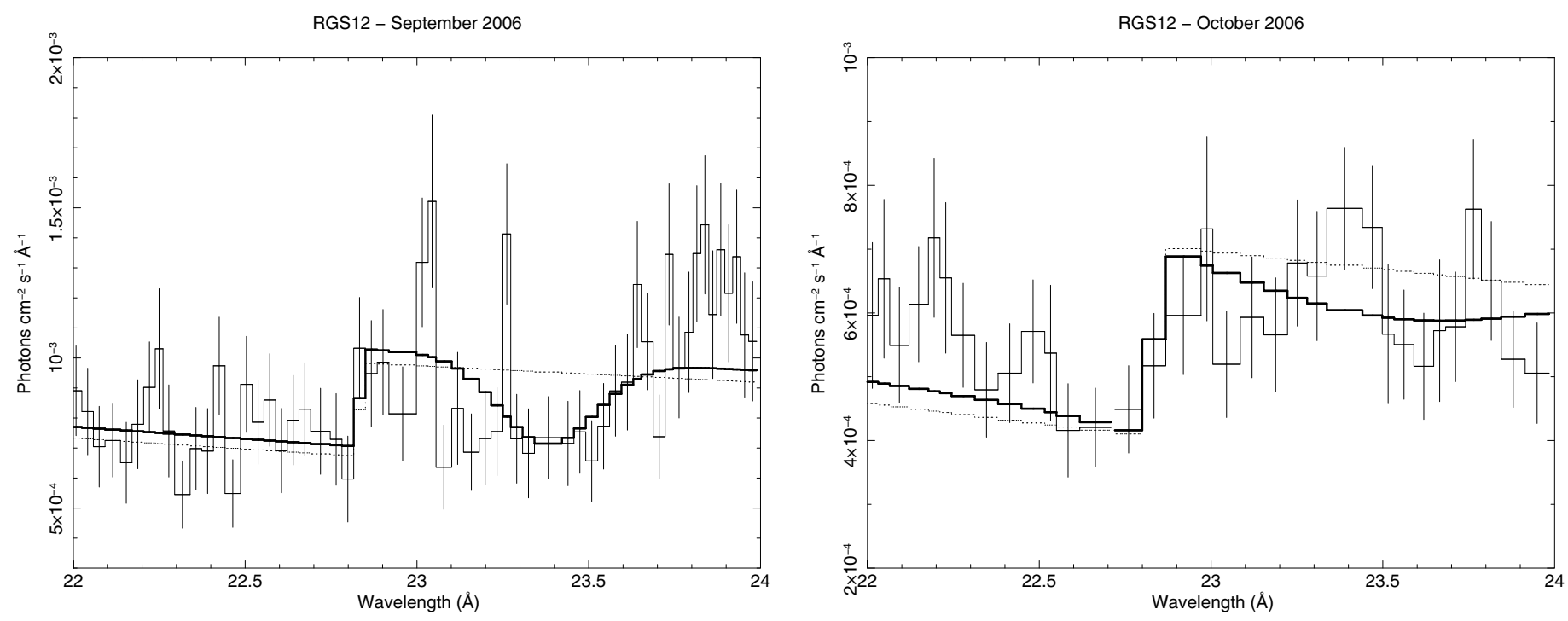

Fig. 4. RGS1 and RGS2 XB 1832-330 spectra in the two observations, limited to the wavelenght region around the Oxygen edge. In the left panel, the excesses just above $23 \AA$ could be due to the O III emission line, but it is less than $2 \sigma$ from the continuum and the statistics hampers a more detailed analysis.

is striking that this period is twice the period found in the first observation performed in 2006 September, within the uncertainties.

\subsection{Spatial analysis: a source catalogue of the XB 1832-330 field}

The EPIC images of the XB 1832-330 field show the presence of several faint X-ray sources. We therefore performed a detection analysis in order to obtain a source catalogue of this region. We considered only the two MOS cameras, since the pn was operated in Small Window mode and could image a region close to the central target.

The cleaned events files were used to produce MOS 1 and MOS2 images in 5 energy ranges: $0.5-1 \mathrm{keV}, 1-2 \mathrm{keV}, 2-5 \mathrm{keV}$, 5-10 keV and 0.5-10 keV (total band). For each energy band, a corresponding set of exposure maps (i.e. one for each detector) was generated with the SAS task expmap, to account for spatial quantum efficiency (QE), mirror-vignetting, and field of view variations. To maximize the signal-to-noise ratio $(S / N)$ of our serendipitous sources and to reach lower flux limits, we merged the data (images and maps) from the two MOS cameras for the two pointings separately. After the production of a detector mask (with the task emask), both images and exposure maps were used as a reference for the source detection, which was performed in four steps:

1. for each of the selected energy bands, the SAS task eboxdetect was run in local mode to create a preliminary source list. Sources were identified by applying the standard minimum detection likelihood criterium, i.e., only candidate sources with detection likelihood $-\ln P \geq 8$ were validated, where $P$ is the probability of a spurious detection due to a Poissonian random fluctuation of the background;

2. then, the task esplinemap was run to remove all the validated sources from the original image and to create a background map by fitting the so-called cheesed image with a cubic spline; 

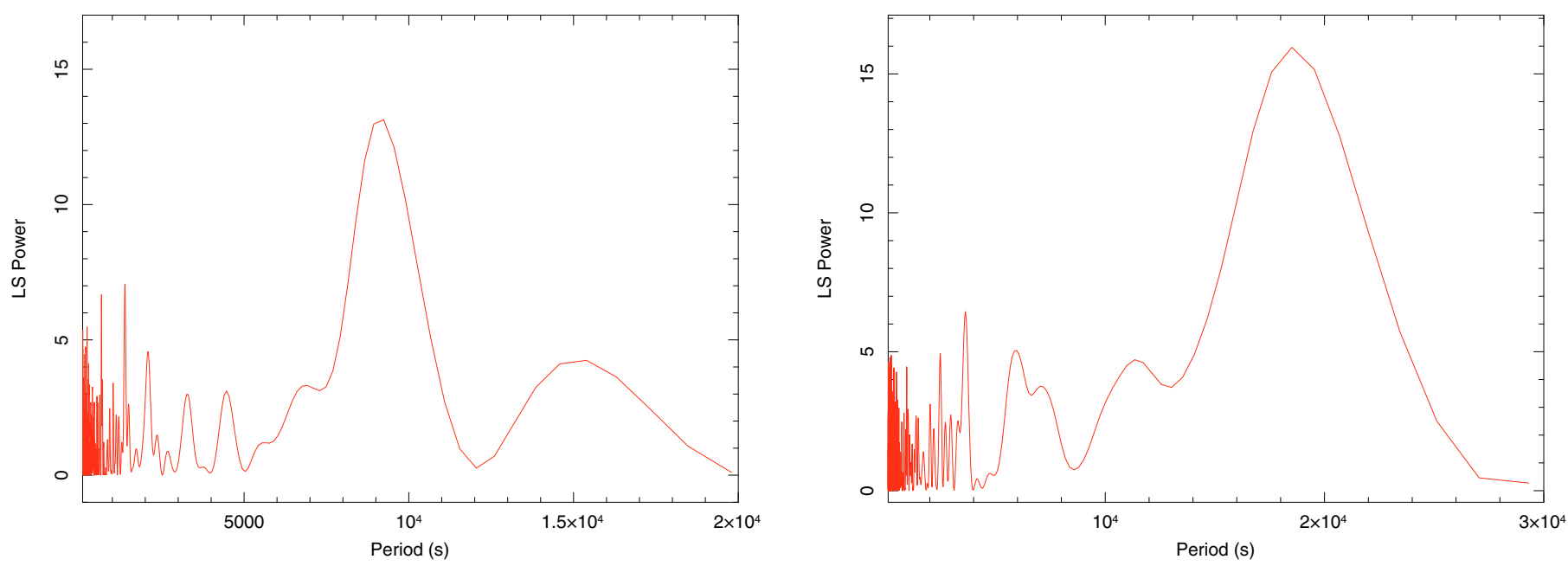

Fig. 5. Lomb-Scargle periodograms of the two XMM-Newton observations, 2006 September on the left and 2006 October on the right.

X1832-330

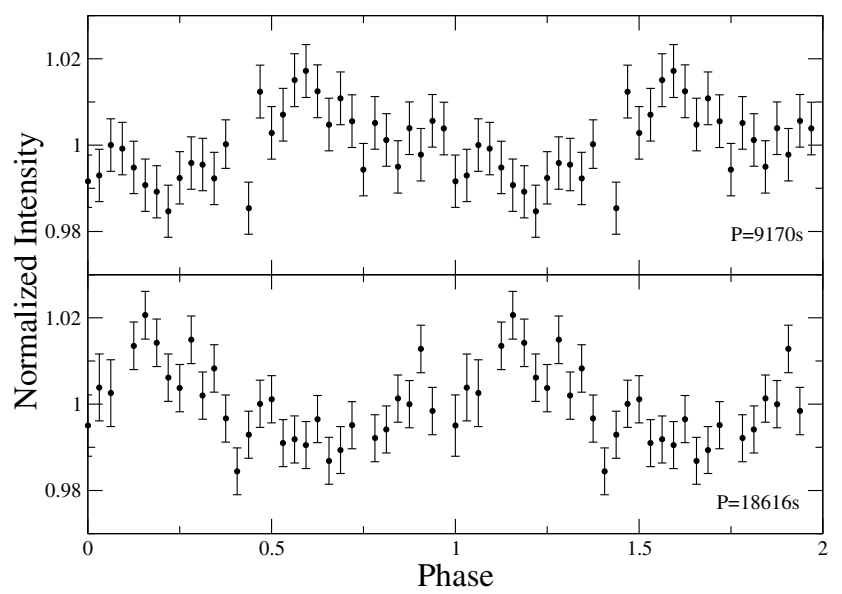

Fig. 6. Lightcurves of the two EPIC observations folded on the periods $P=9170$ s (Obs I, upper panel) and $P=18616$ s (Obs II, lower panel). Phase zero is arbitrary.

3. for each of the selected energy bands, the task eboxdetect was run again, but in map mode using the calculated background maps as a reference;

4. last, the final source positions (with the EPIC combined MOS1+MOS2 count rates in each energy range) were calculated using the task emldetect, which performs maximum likelihood fits to the source's spatial count distribution in all energy bands. In this case, we checked that low threshold values of the equivalent single band detection likelihood (parameter ( $\mathrm{mlmin})$ resulted in many spurious detections. Therefore we fixed $\operatorname{mlmin}=30$, in order to only select the meaningful sources.

With the above procedure we found 25 sources in obs I and 22 in the obs II. To increase the count statistics further, we merged the data (images and maps) of the two pointings and also applied the above detection procedure to the merged data. In this case, we detected a total of 41 sources using the same likelihood limit of $\operatorname{mlmin}=30$.

Finally, we used the srcmatch task to crossmatch the three sets of detected sources to check for coincident ones. This list contains all 46 sources whose distribution over the observed sky area is shown in Fig. 7. There we can check that sources \#15,

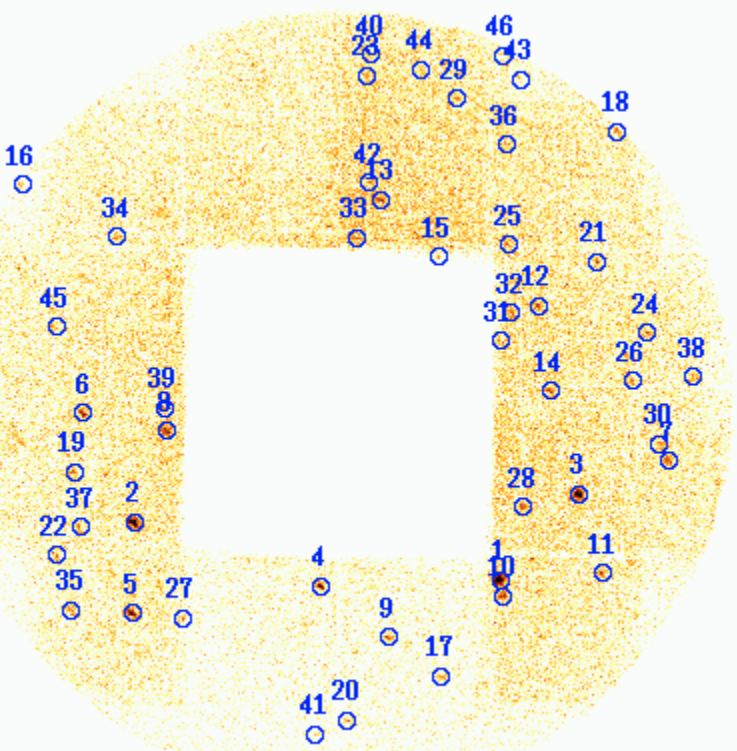

Fig. 7. Distribution of the 46 detected X-ray sources over the EPIC focal plane. The central CCD is empty since it was operated in Small Window mode and no source detection was performed on its data. Numbers mark the 46 sources listed in Table 4.

$\# 16, \# 18$, \#31, and \#46 are close to the edge of the EPIC FOV, so we cannot exclude their being spurious detections.

The main characteristics of these sources are summarised in Table 4. We list the source position (using the recommended XMM-Newton designations for serendipitous sources), the position uncertainty, the count rate in the four fine energy ranges and the two hardness ratios between the two soft and the two hard ranges. In the reported position error we not only consider the statistical errors, associated with the centroiding of the point source, but also the systematic error of $1^{\prime \prime}$ (Kirsch 2007) due to the absolute positional accuracy of XMM-Newton. We also report the total source flux between 0.5 and $10 \mathrm{keV}$, which has been calculated from the total count rate in the full energy band. The applicable count rate-to-flux conversion factor has been calculated assuming a power-law spectrum with photon index, $\Gamma$, of 2 
and the hydrogen column density in the direction of the globular cluster.

For each source we report whether it was detected in the data of the single and/or the combined observations. In this way we find that 11 sources of the first observation are not detected in the second one, while 8 sources of the second observation are not detected in the first one. To verify that this result is due to a real source variability, we analysed both the position and count rates of these sources. We find that, in most cases, a source is detected in only one observation because in the other it either falls out of the imaged field-of-view (since the two observations are slightly rotated) or its count rate is just below the detection limit, even if the count rates measured in both the observations are consistent at a $1 \sigma$ confidence level. Only in a few cases (highlighted with a "V" in Table 4) we checked that the missing detection is due to a real source variability. We find that only 14 sources detected in the merged observations (over a total of 41) are also detected in both the individual observations, while 8 and 6 are only detected in obs I and II, respectively; and the remaining 13 sources are detected neither in the first nor the second observation. Finally, we also note that 3 sources of obs I and 2 sources of the obs II are not detected in the merged observation.

We searched for possible known counterparts in the SIMBAD database, assuming an uncertainty radius equal to three times the source positional uncertainty. In this way we found no positionally coincident sources. The object known as NGC 6652 10, discovered by ROSAT (Johnston et al. 1996), is at a distance of $10.8^{\prime \prime}$ from our source \#4. In Fig. 7 we note that this source is rather bright and, moreover, that there are no other $\mathrm{X}$-ray sources near its position. Therefore, we suggest that it is indeed NGC 6652 10, whose position is now better constrained by the XMM-Newton measurements.

With the same search criteria, we searched for possible counterparts in the GSC2.3 (McLean et al. 2000), USNO-NOMAD (Monet et al. 2003), and 2MASS (Cutri et al. 2003) catalogues. As shown in Table 4, with these constraints we found candidate counterparts for only 10 sources. To evaluate whether they are due to possible foreground contamination, we have to estimate the probability of a chance coincidence between an X-ray and an optical source. This parameter is given by $P=1-\mathrm{e}^{-\pi r^{2} \mu}$, where $r$ is the X-ray uncertainty-circle radius and $\mu$ is the surface density of the optical sources (Severgnini et al. 2005). In our case, the surface density of the GSC2.3, USNO-NOMAD and 2MASS sources in the EPIC field-of-view is $7.3 \times 10^{-3}, 1.7 \times 10^{-2}$, and $3.6 \times 10^{-3} \operatorname{arcsec}^{-2}$, respectively. Based on the estimated position errors, we derived $P>10 \%$ for all the candidate counterparts; therefore, we cannot exclude that they are due to random coincidences.

\section{Discussion}

We report here on two XMM-Newton observations of XB 1832-330 (located in NGC 6652) performed in 2006, about 1 month apart, which are the first high-resolution spectra of this globular cluster source. XB 1832-330 is one of the luminous LMXBs $\left(L_{X}>10^{36} \mathrm{erg} \mathrm{s}^{-1}\right)$ located in a galactic globular cluster, and it is supposed to be an ultracompact binary, because of the faintness of its optical counterpart (Heinke et al. 2001). Ultracompactness is also suggested by one of the possible periodicities found in optical data (Heinke et al. 2001), if associated with the orbital period.

The $0.3-12 \mathrm{keV}$ spectrum is complex and cannot be fit by a single model, requiring a soft component (described here by a blackbody with a temperature of $0.6 \mathrm{keV}$ ), together with a hard power law (photon index of $\sim 1.5$ ). The spectral parameters of the soft component are similar to those obtained by Parmar et al. (2001) during a BeppoSAX observation. The source also displays a similar 1-10 keV luminosity (with XMM-Newton we get $\sim 1.4 \times 10^{36} \mathrm{erg} \mathrm{s}^{-1}$ in the same energy range). The XMM-Newton spectroscopy is compatible with what was found by Sidoli et al. (2001) in ultracompact binaries located in galactic globular clusters. Indeed, these sources have quite different properties from all the other bright globular cluster LMXBs with longer orbital periods. This spectral analogy led Parmar et al. (2001) to suggest that XB 1832-330 could be an ultracompact binary.

The total low-energy absorption resulting from the fits is compatible with the optically derived value in the direction of the host globular cluster of $5.5 \times 10^{20} \mathrm{~cm}^{-2}$ in the first observation, while it is slightly higher in the second, suggesting an extra-absorption in the line of sight of $\sim 3 \times 10^{20} \mathrm{~cm}^{-2}$. The likely presence of intrinsic neutral extra-absorption seems to be confirmed by a good fit when using the partial covering fractional absorption model, which indicates that XB 1832-330 is absorbed by an additional neutral medium with a covering factor of $\sim 30 \%$ and an intrinsic hydrogen column density in the range $3-4 \times 10^{21} \mathrm{~cm}^{-2}$ for the two observations.

From the analysis of ASCA spectra of a few ultracompact binaries, Juett et al. (2001) suggested the presence of an excess absorption of neutral Ne-rich material local to the sources, leading some authors to propose that the donor stars in some ultracompact binary systems are Ne-rich white dwarfs (e.g., Yungelson et al. 2002). If this is the case, the high $\mathrm{Ne} / \mathrm{O}$ ratio could be a spectral signature of the presence of some types of degenerate companions (e.g. neon-rich $\mathrm{CO}$ white dwarf or $\mathrm{O}-\mathrm{Ne}-\mathrm{Mg}$ WD donors). High-resolution spectra taken with XMM-Newton and Chandra confirmed a neutral Ne overabundance in a few cases (e.g., 4U 0614+091, Paerels et al. 2001; 4U 1543-624 and 2S 0918-549, Juett \& Chakrabarty 2003). Instead, in other confirmed ultracompact sources, an overabundance of neutral $\mathrm{Ne}$ has not been found (e.g. 4U 1850-087, Sidoli et al. 2005; and Juett \& Chakrabarty 2005) with high-resolution spectra, contrary to the earlier ASCA measurement (Juett et al. 2001). This indicates a variability in the $\mathrm{Ne} / \mathrm{O}$ ratio probably due to luminosity variations (see e.g. Juett \& Chakrabarty 2003; or Sidoli et al. 2005). On the other hand, several known ultracompact binaries have never shown evidence of unusual $\mathrm{Ne} / \mathrm{O}$ ratios (e.g. $4 \mathrm{U}$ 1820-30, where an He white dwarf donor is present, or 4U 0513-40, Juett \& Chakrabarty 2005).

We adopted a variable absorption model, together with three edges corresponding to $\mathrm{O}-\mathrm{K}, \mathrm{Ne}-\mathrm{K}$ and $\mathrm{Fe}-\mathrm{L}$ features in order to measure the column density of the neutral $\mathrm{Ne}, \mathrm{O}$, and $\mathrm{Fe}$ in the line of sight. The high-resolution XB 1832-330 spectra presented here do not show any prominent emission features. Our study of the strength of the edges of neutral $\mathrm{Ne}$ and $\mathrm{O}$ reveals an $\mathrm{Ne} / \mathrm{O}$ abundance ratio (see Table 3), which is consistent with the ISM value of 0.18 (Wilms et al. 2000). This implies that there is no evidence of the donor star in XB 1832-330 being an Ne-enriched white dwarf.

If we compare the measured elemental column densities $N_{\mathrm{Z}}$ (converted to equivalent $\mathrm{H}$ column densities using the Wilms et al. (2000) ISM abundances) with the $N_{\mathrm{H}}$ resulting from the overall shape of the XMM-Newton spectra, there seems to be a discrepancy, which could indicate an over-abundance of $\mathrm{O}$ and a sub-solar abundance of Fe. This can be simply explained by the low $\mathrm{Fe}$ abundance of the globular cluster. We note that we did not assume any uncertainty in the photoelectric 


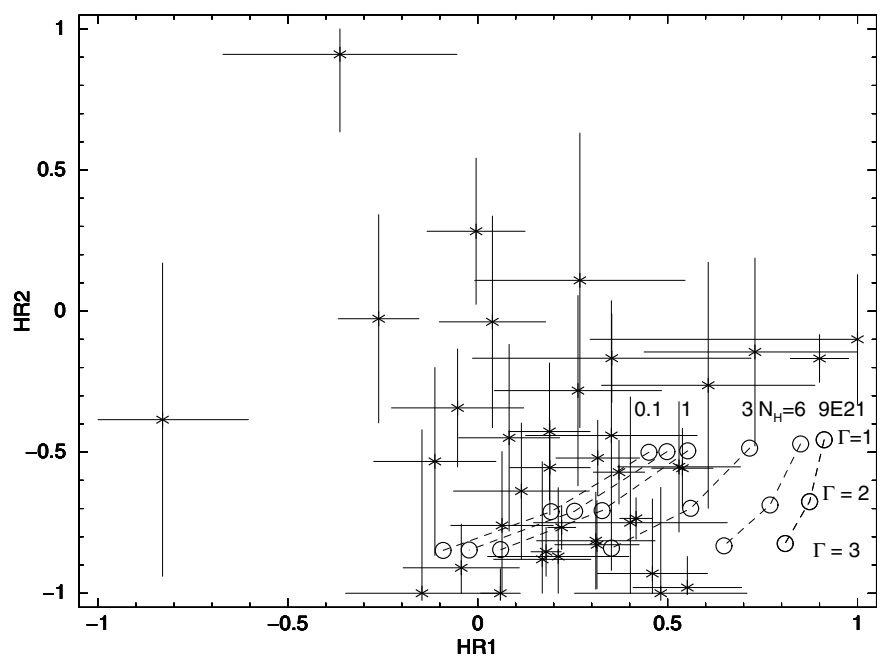

Fig. 8. Colour-colour diagram of the detected sources. Open circles mark the simulated colours (obtained with an absorbed power-law spectrum, assuming different photon indexes and absorption columns). From top to bottom the photon index is 1,2 , and 3; from left to right different column densities have been assumed: $0.1,0.5,1,3,6,9 \times 10^{21} \mathrm{~cm}^{-2}$.

cross-sections, which could be affected by an uncertainty as large as 30\% (Paerels et al. 2001). This could account for the observed discrepancy. On the other hand, the resulting column density is also dependent on the model adopted for the continuum. A Comptonization model, for example, leads to a lower absorption, because of the roll-over at soft energies.

The timing analysis resulted in a possible periodicity of $\sim 2.5 \mathrm{~h}$ in the EPIC lightcurve of the first observation and in a second periodicity (about twice what is found in Obs I) during Obs II. The smallest period, if linked to the orbital period of the binary system, would exclude an ultracompact binary (where orbital periods are less than about $1 \mathrm{~h}$ ). We note, however, that, although the period is significant due to the limited duration of the observations, it is not possible to clearly say if this variability is a stable feature in the X-ray lightcurve or if it is a transient effect not linked to the orbital motion.

Our spatial analysis has led to the compilation of a catalogue of 46 faint X-ray sources in the XB 1832-330 field, most of which probably do not belong to the globular cluster, as the NGC 6652 tidal radius is $4.48^{\prime}$ (the 2003 version of the catalogue is described in Harris 1996), well inside the central CCD of the MOS cameras, which was not used for source detection. These sources are not listed in the second XMM-Newton (2XMM) Serendipitous Source Catalogue (available online at
http://xmmssc-wWw.star. le.ac.uk/Catalogue/2XMM). In Fig. 8 we report the colour-colour diagram of the detected sources. For comparison, we overplot the simulated colours obtained with an absorbed power-law spectrum, assuming different photon indices and absorption columns. Most of the sources display a low absorption $\left(\sim 10^{20} \mathrm{~cm}^{-2}\right)$, lower than the absorption towards NGC 6652, making them likely foreground objects.

Acknowledgements. Based on observations with XMM-Newton, an ESA science mission with instruments and contributions directly funded by ESA member states and the USA (NASA). This work was supported by contract ASI/INAF I/023/05/0 and ASI/INAF I/008/07/0. We thank the referee (A. Juett) for constructive comments and suggestions which significantly improved our paper.

\section{References}

Boirin, L., Méndez, M., Díaz Trigo, M., Parmar, A. N., \& Kaastra, J. S. 2005, A\&A, 436, 195

Cutri, R. M., Skrutskie, M. F., van Dyk, S., et al. 2003, VizieR Online Data Catalog, 2246

Harris, W. E. 1996, AJ, 112, 1487

Heinke, C. O., Edmonds, P. D., \& Grindlay, J. E. 2001, ApJ, 562, 363

Henke, B. L., Gullikson, E. M., \& Davis, J. C. 1993, Atomic Data and Nuclear Data Tables, 54, 181

Hertz, P., \& Wood, K. S. 1985, ApJ, 290, 171

in 't Zand, J. J. M., Verbunt, F., Heise, J., et al. 1998, A\&A, 329, L37

Johnston, H. M., Verbunt, F., \& Hasinger, G. 1996, A\&A, 309, 116

Juett, A. M., \& Chakrabarty, D. 2003, ApJ, 599, 498

Juett, A. M., \& Chakrabarty, D. 2005, ApJ, 627, 926

Juett, A. M., Psaltis, D., \& Chakrabarty, D. 2001, ApJ, 560, L59

Juett, A. M., Schulz, N. S., \& Chakrabarty, D. 2004, ApJ, 612, 308

Kirsch, M. 2007 ,

http://xmm.vilspa.esa.es/docs/documents/CAL-TN-0018.pdf

McLean, B. J., Greene, G. R., Lattanzi, M. G., \& Pirenne, B. 2000, in Astronomical Data Analysis Software and Systems IX, ed. N. Manset, C. Veillet, \& D. Crabtree, ASP Conf. Ser., 216, 145

Monet, D. G., Levine, S. E., Canzian, B., et al. 2003, AJ, 125, 984

Mukai, K., \& Smale, A. P. 2000, ApJ, 533, 352

Ortolani, S., Bica, E., \& Barbuy, B. 1994, A\&A, 286, 444

Paerels, F., Brinkman, A. C., van der Meer, R. L. J., et al. 2001, ApJ, 546, 338

Parmar, A. N., Oosterbroek, T., Sidoli, L., Stella, L., \& Frontera, F. 2001, A\&A, 380,490

Predehl, P., \& Schmitt, J. H. M. M. 1995, A\&A, 293, 889

Predehl, P., Hasinger, G., \& Verbunt, F. 1991, A\&A, 246, L21

Severgnini, P., Della Ceca, R., Braito, V., et al. 2005, A\&A, 431, 87

Sidoli, L., Parmar, A. N., Oosterbroek, T., et al. 2001, A\&A, 368, 451

Sidoli, L., La Palombara, N., Oosterbroek, T., \& Parmar, A. N. 2005, A\&A, 443, 223

Tarana, A., Bazzano, A., Ubertini, P., \& Federici, M. 2006, [arXiv: astro-ph/0610325]

Wilms, J., Allen, A., \& McCray, R. 2000, ApJ, 542, 914

Yungelson, L. R., Nelemans, G., \& van den Heuvel, E. P. J. 2002, A\&A, 388, 546 


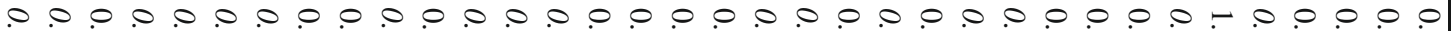

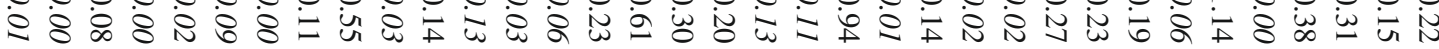

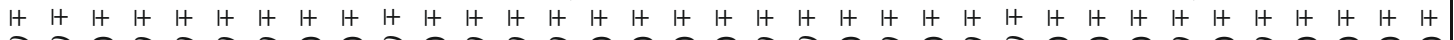

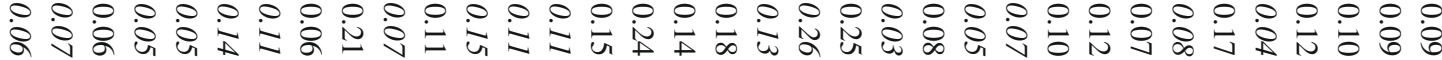

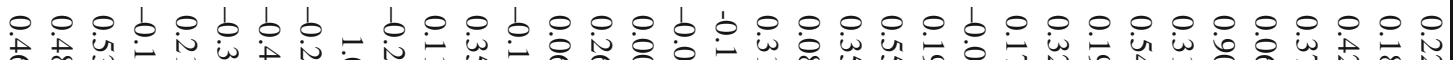

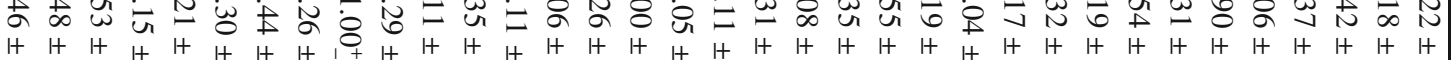

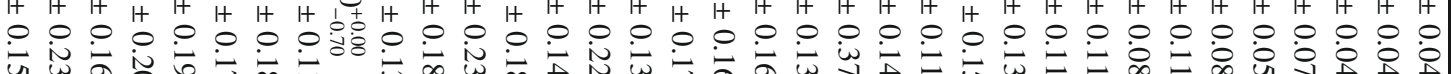
U wa w w w

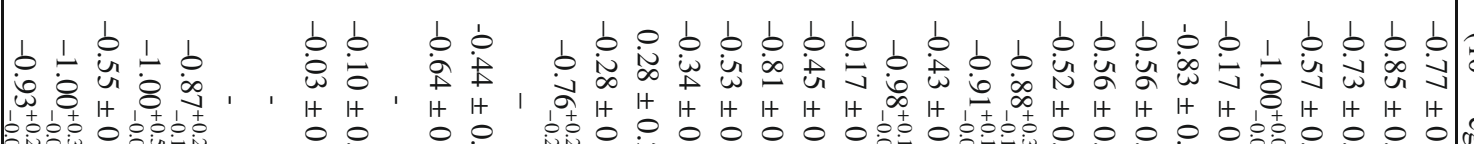

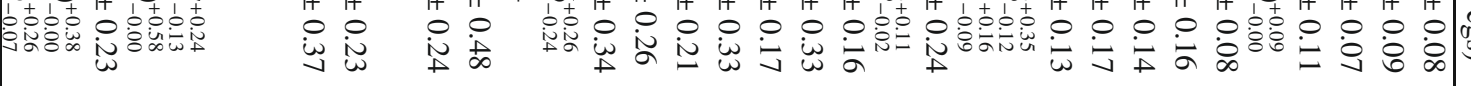

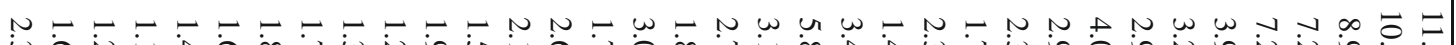

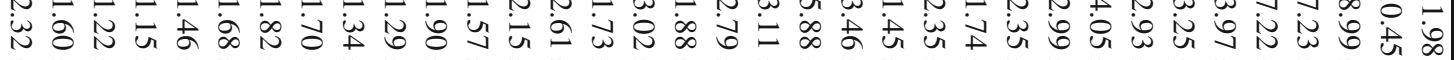

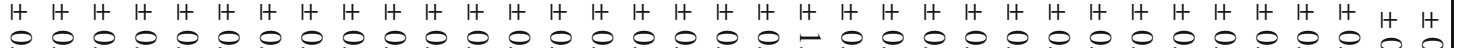

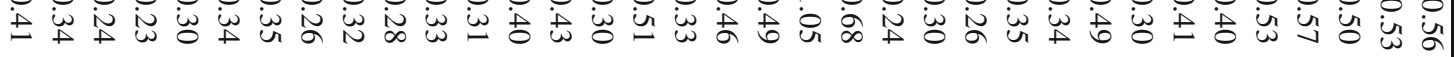

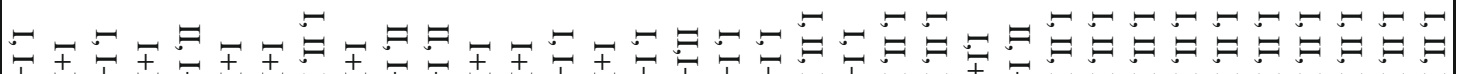

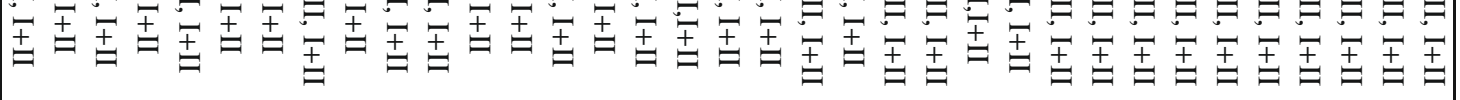

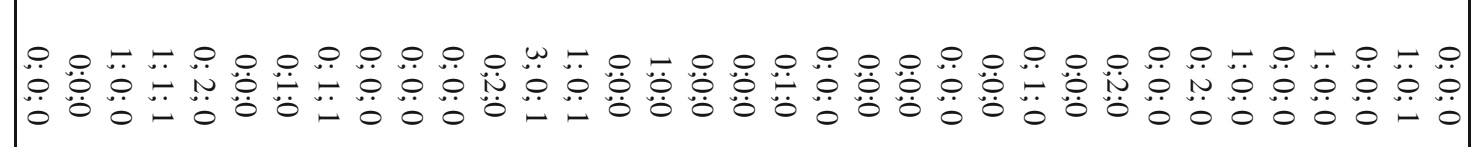


L. Sidoli et al.: XMM-Newton observations of XB 1832-330 in NGC 6652, Online Material p 2

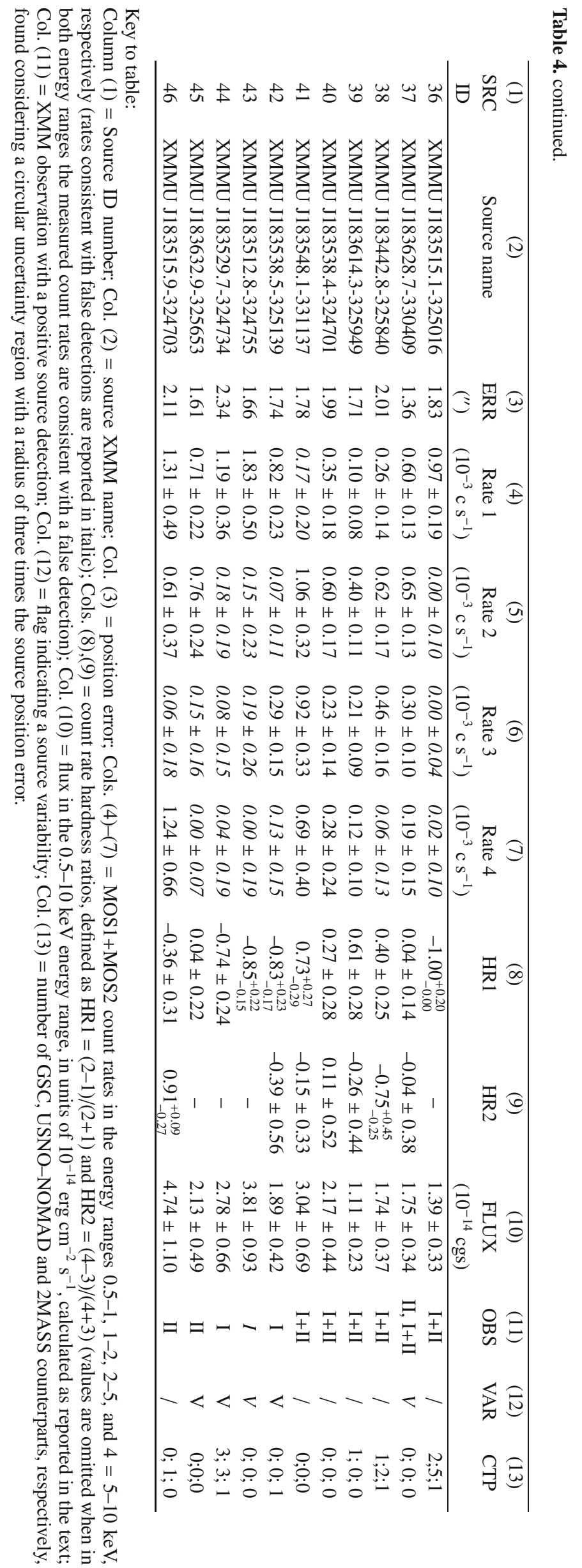

